\title{
Gout: Update on Current Therapeutics
}

Sneha Pai, MD

Ashraf Raslan, MD

Naomi Schlesinger, $M D^{*}$

\author{
Address \\ *Division of Rheumatology, Department of Medicine, Rutgers-Robert Wood Johnson \\ Medical School, MEB 468, P0 Box 19, New Brunswick, NJ 08903-0019, USA \\ Email: schlesna@rwjms.rutgers.edu
}

Published online: 10 March 2015

(C) Springer International Publishing AG 2015

This article is part of the Topical Collection on Gout

Keywords Acute gout treatment · Gout prophylaxis · Urate-lowering therapy

\section{Opinion statement}

Gout is the most common inflammatory arthritis in adults. Given the increasing prevalence of gout and associated comorbidities, there is a growing need for better therapeutic options. While current treatment options satisfactorily control the disease in some patients, many patients, especially those with associated comorbidities, have contraindications to current treatments and need more treatment options. New drugs are needed to further improve our treatment, especially in patients with associated comorbidities. The duration of therapy for acute and chronic gout as well as combination of therapies, recommended by the American College of Rheumatology (ACR) guidelines, need to be further studied. The past decade has been an exciting time for gout research with significant advances in our understanding of disease pathogenesis and its treatment. The new ACR guidelines have improved gout visibility and will improve gout awareness and treatment in the future. With growing treatment options, gout is becoming a disease that can be cured.

\section{Introduction}

In 1964, Dr. Talbot wrote that "the centuries-old recognition of gout as a clinical entity has tended to cause traditional therapeutics measures mainly unsatisfactory, to be handed down form teacher to pupil with only slight modification through the years" [1]. Over the past 10 years, gout treatment has advanced greatly. However, in a growing number of patients, current treatments may still be unsatisfactory. Hypertension, cardiovascular disease, diabetes, renal impairment, peptic ulcer disease, the metabolic syndrome, and liver disease are highly prevalent in gout patients and may lead to standard treatments being contraindicated. Currently, there are formal guidelines and recommendations, addressing some of these issues, for the treatment of gout. In this paper, new and old treatment options available for the treatment of acute and chronic gout are reviewed. 


\section{Acute gout}

The current available treatment options in the USA for acute gout are non-steroidal anti-inflammatory drugs (NSAIDs), colchicine, systemic and intra-articular corticosteroids (CS), and adrenocorticotrophic hormone (ACTH). The most important determinant of therapeutic success is how soon the treatment is initiated that the dose chosen be sufficient and for that it be given for a long enough period of time. The treatment choice should be based on the number of joints involved and, thus, the severity of the attack, underlying comorbidities, and medications the patient is on.

Monotherapy may be more appropriate when only a few joints are involved and the attack is considered mild to moderate. NSAIDs and colchicine are firstline drugs for acute attacks. The relative efficacy of colchicine compared with NSAIDs is unknown. Combination therapy may be appropriate when the attack is polyarticular, considered severe or not responding to monotherapy. If the patient is on urate-lowering therapy (ULT) at the onset of the attack, treatment with ULT should not be interrupted nor should the ULT dose be changed during the acute attack.

Colchicine is one of the oldest medications used to treat acute gout and has been widely used; however, only in 2009 was it approved by the Food and Drug Administration (FDA) for use in acute gout. Colchicine efficacy is derived from modulation of several anti-inflammatory pathways associated with gout [2]. It binds to tubulin dimers, thus preventing microtubule assembly and thereby disrupts the NACHT, LRR, and PYD domaincontaining protein 3 (NALP3) inflammasome-driven caspase-1 activation and IL-1 $\beta$ processing, microtubule-based inflammatory cell chemotaxis, generation of leukotrienes and cytokines, and phagocytosis. The American College of Rheumatology (ACR) task force panel (TFP) suggested that colchicine be considered an appropriate first-line treatment for acute gout when colchicine is initiated within $36 \mathrm{~h}$ or less from the onset of the attack [3•]. The recommended loading dose is $1.2 \mathrm{mg}$ followed by $0.6 \mathrm{mg}$ after $1 \mathrm{~h}$. This recommendation is based on the recently published Acute Gout Flare Receiving Colchicine Evaluation (AGREE) trial [4]. One hundred and eighty-four patients with acute attacks of less than 12-h duration were enrolled in a study that compared low-dose colchicine, high-dose colchicine ( $4.8 \mathrm{mg}$ total over $6 \mathrm{~h}$ ), and placebo. The primary endpoint was a $50 \%$ reduction or greater in pain within $24 \mathrm{~h}$ of enrollment. Low-dose colchicine resulted in a similar efficacy with fewer side effects as compared to high-dose colchicine [4]. The most common side effects reported with oral colchicine in clinical trials were diarrhea, nausea, vomiting, and nasopharyngeal pain $[4,5]$. These side effects were considered mild and resolved with dose reduction. More severe side effects were observed with overdoses of colchicine, including bone marrow suppression with agranulocytosis. 
Colchicine is metabolized by the cytochrome P450 (CYP) 3A4 enzyme and is a substrate for the P-glycoprotein 1 (P-gp) efflux transporter [6]. Caution is advised when colchicine is used in patients with hepatic or renal impairment, and dose should be adjusted according to the degree of impairment. Concurrent use of interacting drugs or treatment with colchicine in patients with renal impairment has been associated with myopathy or rhabdomyolysis [7]. Colchicine administration is contraindicated in patients with renal or hepatic impairment receiving both P-gp and strong CYP3A4 inhibitors concurrently (http://www.drugs.com/pro/colcrys.html).

\section{NSAIDs}

NSAIDs are considered a first-line therapy for acute gout attacks. Historically, indomethacin was the NSAID of choice for the treatment of acute gout. In headto-head studies though, the different NSAIDs have similar efficacy in treating acute gout [8]. Selective cyclooxygenase (COX)-2 inhibitors and non-selective NSAIDs have the same efficacy with the advantage that the COX-2 inhibitors are associated with significantly fewer adverse events [8]. The NSAIDs currently approved by the FDA for the treatment of acute gout include naproxen, indomethacin, and sulindac. Thus, the selective COX-2 inhibitors are not currently approved by the FDA for the treatment of acute gout. NSAIDs should be avoided in patients with active or recent peptic ulcer disease and in patients with renal insufficiency.

CS are recommended as a first-line treatment option for acute gout [3•], especially when the use of NSAIDs and/or colchicine is not efficacious, poorly tolerated, or contraindicated. CS may be a safer treatment option for patients with acute gout who have an underlying renal impairment, a common comorbidity in gout patients.

Monoarticular attacks are often managed with the use of intra-articular (IA) CS. However, evidence for the use of IA and intramuscular (IM) CS is limited. Treatment with IA CS was recommended by the ACR TFP [3•] when only one or two large joints were involved. The use of systemic CS was recommended in all other gout attacks, where there is polyarticular joint involvement or when joint injections are not feasible.

In two randomized, placebo-controlled trials of a 5-day course of oral prednisolone (one evaluating a dose of $30 \mathrm{mg}$ daily and the other a dose of $35 \mathrm{mg}$ daily), the efficacy of prednisolone $30 \mathrm{mg}$ daily was equivalent to that of standard regimens of indomethacin and naproxen versus prednisolone 35 daily $[9 \bullet, 10]$. The ACR TFP recommended that prednisone or prednisolone be started at a dose $\geq 0.5 \mathrm{mg} / \mathrm{kg}$ daily for 510 days followed by discontinuation or alternately 2-5 days at the full dose followed by dose tapering over 7-10 days and then discontinuation $[3 \bullet]$.

The TFP [3•] also recommended an alternative regimen of an IM single dose (60 mg) of triamcinolone acetonide, followed by oral prednisone or prednisolone [11]. However, there was no consensus by the TFP on the use of IM triamcinolone acetonide as monotherapy. 


\section{ACTH}

ACTH has long been used in acute gout [12]. ACTH stimulates the adrenal cortex to produce CS and interferes with the acute inflammatory response through the activation of the melanocortin receptor-3.

A recent retrospective study of 181 in-patients who received IM ACTH for their acute gout attack found that most of them (78\%) had a significant response to the treatment and all responded to a repeated single dose of ACTH [13]. The ACR TFP recommended that ACTH can be used for the treatment of acute attacks with a single IM injection ( 25 to $40 \mathrm{IU}$ ) which they suggested that it may be repeated every $24-72 \mathrm{~h}$ as clinically indicated [3•]. ACTH is not approved by FDA for the treatment of acute gout. ACTH is very expensive in the USA, which limits its use for the treatment of acute gout.

\section{Investigational drugs}

\section{IL-1 $\beta$ inhibitors}

NACHT, LRR, and PYD domain-containing protein 3 (NALP3) inflammasome activation and release of interleukin-1 (IL-1) play a central role in the initiation of the inflammatory response to monosodium urate (MSU) crystals [14•]. IL-1 inhibitors being tested for gout include anakinra, rilonacept, and canakinumab.

Anakinra is a recombinant human IL-1 receptor antagonist (IL-1Ra) with a short half-life of 4-6 h. So et al. reported significant pain relief in 10 gout patients with acute or subacute gout who could not tolerate or had failed standard antiinflammatory therapies for acute gout in when anakinra $100 \mathrm{mg}$ daily was administered subcutaneously (SC) for 3 days [15]. In a retrospective study [16] of 15 hospitalized patients who had failed CS treatment or had comorbid limitations to the use of standard anti-inflammatory therapies for acute gout, anakinra was successful in resolving acute gout attacks in all patients.

Rilonacept, an IL-1 trap with a half-life of approximately 8.6 days, was found to decrease pain in patients with chronic active gout when 160-mg SC was administered once weekly [17]. However, when rilonacept was combined with indomethacin and when used alone, it failed to improve pain relative to indomethacin during acute gout [18].

Canakinumab is a monoclonal anti-human IL-1 $\beta$ antibody with a long half-life of approximately 28 days. In an acute gout study, one SC injection of canakinumab $150 \mathrm{mg}$ in patients who were refractory to or had a contraindications to NSAIDs and/or colchicine [19] found canakinumab to be superior, starting at $24 \mathrm{~h}$, in providing pain relief compared to $40 \mathrm{mg}$ of triamcinolone acetonide given IM. The median time to $50 \%$ reduction in pain was reached at one day in patients treated with SC canakinumab $150 \mathrm{mg}$ versus 2 days for patients treated with IM triamcinolone acetonide $(P=0.0006)$ [19].

\section{Non-pharmacological treatment}

\section{Topical ice}

Cold applications may be a useful adjunct to treatment of acute gout. In a small prospective randomized trial, patients with acute gout treated with topical ice 
had a greater reduction in pain $(P=0.021)$, joint circumference, and synovial fluid volume compared with the control group not treated with topical ice [20].

\section{Chronic gout}

\section{Prophylaxis}

Prophylaxis refers to chronic treatment with anti-inflammatory drugs in order to prevent gout attacks. Persistent low-grade inflammation is frequently present in asymptomatic chronic tophaceous gout. Even when the patient is asymptomatic, chronic inflammation is often present in patient [21]. In addition, it has been observed that when ULT is initiated, there is an increase in acute attacks. The association between initiation of ULT and acute attacks has been confirmed by results of the febuxostat [22] and pegloticase trials [23].

\section{Colchicine}

Colchicine has been used for gout prophylaxis for many years, and studies have demonstrated the efficacy of prophylactic colchicine for the reduction or prevention of attacks upon ULT initiation $[5,22,24]$. However, it was not approved by the US FDA for this indication until 2009 [25]. Colchicine is currently considered to be the standard of care for attack prophylaxis during initiation of ULT $[3 \bullet, 26]$ and is currently the only FDA-approved treatment for gout prophylaxis.

The tolerability profile of colchicine is dose dependent, and the recommended dose of colchicine prophylaxis (0.6 mg once or twice daily) [3•] is generally well tolerated, with diarrhea as the most commonly reported adverse event that may resolve when dosage is reduced to a once-daily administration instead of twice daily [5]. Dose reduction is recommended for patients with chronic kidney disease (CKD) [26] and when co-administered with drugs known to inhibit cytochrome P450 3A4 (CYP3A4), particularly clarithromycin CYP3A4 and/or Pglycoprotein, due to the increased risk of colchicine-induced toxic effects [27].

\section{NSAIDs}

NSAIDs are used less commonly used for prophylaxis during initiation of ULT than colchicine. Controlled studies evaluating their efficacy and safety in this population are limited [28]. NSAIDs are not ideal for prophylaxis since many gout patients have contraindications to NSAIDs use such as renal impairment and poorly controlled hypertension [29]. In addition, low-dose NSAIDs, such as aspirin, can decrease uricosuria; therefore, increasing serum urate (SU) levels and chronic NSAID use can increase the incidence of gastrointestinal events as well as cardiovascular risks. A careful assessment for such comorbidities should be undertaken before NSAIDs are used for prophylaxis.

\section{Investigational drugs}

IL-1 inhibition may be beneficial as gout prophylaxis, in addition to its role in the treatment of acute gout [30]. Rilonacept [31] and canakinumab [32•] have been studied as prophylactic drugs when initiating ULT. 
Rilonacept was studied in 83 patients initiating allopurinol $300 \mathrm{mg}$ daily [29] randomized to 16 weeks of placebo versus weekly SC rilonacept $160 \mathrm{mg}$. Rilonacept prophylaxis markedly reduced the frequency of attacks. At week 16, the occurrence of attacks was significantly reduced with rilonacept compared with placebo $(0.22$ vs $0.93 ; P=0.0036)$ and the percentage of patients who experienced $\geq 1$ attack was significantly lower (22 vs $48 \% ; P=0.0209)$. In the canakinumab prophylaxis trial [32•], 432 patients initiating ULT with allopurinol were enrolled. Over 16 weeks, there was a 64 to $72 \%$ reduction in the risk of experiencing $\geq 1$ attack for canakinumab doses $\geq 50 \mathrm{mg}$ versus colchicine $(P \leq 0.05)$, and the percentage of patients who experienced $\geq 1$ attack was significantly lower ( 15 to $27 \%$ vs $44 \%$; $P<0.05$ ).

\section{Non-pharmacological treatment}

Cherry consumption is efficacious for the prevention of gout attacks [33]. Gouty inflammation is primarily interleukin 1 (IL-1) mediated [34]. In-vitro cherry juice concentrate can reduce the release of IL-1 by monocytes activated by monosodium urate crystals. Consumption of cherry juice concentrate reduces acute gout attacks when taken over a period of 4 months or longer [35]. Cherry juice concentrate is suspected to have anti-inflammatory properties and may be useful for gout prophylaxis.

Urate-lowering therapy

Hyperuricemia is the hallmark of gout. Hyperuricemia is defined as a serum urate (SU) level $\geq 6.8 \mathrm{mg} / \mathrm{dl}$ which corresponds to the physiologic saturation threshold of urate [21]. A SU level $\geq 6.8 \mathrm{mg} / \mathrm{dl}$ facilitates precipitation of MSU crystals. The 2012 ACR guidelines [36•] recommended the use of xanthine oxidase inhibitors (either allopurinol or febuxostat) as first-line therapy.

\section{Xanthine oxidase inhibitors (XOI)}

Xanthine oxidase $(\mathrm{XO})$ is the rate-limiting enzyme in the synthesis of uric acid, and hence, inhibition of this enzyme decreases urate synthesis.

Allopurinol was approved by the FDA in 1966 for the treatment of gout. Physicians have gained comfort prescribing allopurinol up to $300 \mathrm{mg} /$ day despite its approval by the FDA in doses up to $800 \mathrm{mg} /$ day. However, at this dose, allopurinol monotherapy is many times insufficient to attain target SU of $<6 \mathrm{mg} / \mathrm{dl}$ [37]. The ACR TFP [36•] recommended allopurinol as a first-line urate-lowering therapy. A Cochrane review analyzed the efficacy of allopurinol compared to other ULTs. The meta-analysis included 11 studies [38]. The incidence of side effects was similar when allopurinol (100$600 \mathrm{mg} /$ day) was compared with placebo, benzbromarone (100-200 mg/ day), and febuxostat ( $80 \mathrm{mg} /$ day). Allopurinol was more efficacious than placebo but less efficacious than febuxostat in achieving a target SU level of $<6 \mathrm{mg} / \mathrm{dl}$.

Allopurinol is generally safe. Rarely, patients develop the lifethreatening allopurinol hypersensitivity reaction. Patients at high risk for these reactions include the Han Chinese, Thai descent, and Koreans with 
stage 3 or worse CKD. In these patients, HLA-B*5801 allele testing prior to initiation of allopurinol is recommended [36•]. It is advisable to start allopurinol at a dose of $100 \mathrm{mg} /$ day in patients with normal renal function and at $50 \mathrm{mg} /$ day in patients with moderate to severe CKD. The ACR TFP [36•] suggests gradual up-titration to doses of $300 \mathrm{mg} /$ day and higher, even in the presence of CKD.

Febuxostat was FDA approved in 2009 and is an important alternative for patients who are intolerant/contraindicated or refractory to other ULT. The FDA-approved doses in the USA are $40-80 \mathrm{mg} /$ day. The Cochrane review [38] reported a $40 \mathrm{mg} /$ day dose of febuxostat to have similar efficacy to that of $300 \mathrm{mg} /$ day of allopurinol, while higher doses $(80 \mathrm{mg} /$ day $)$ of febuxostat were found to be more efficacious in getting to SU target. The CONFIRMS trial compared the efficacy to reduce SU of two doses febuxostat (40 and $80 \mathrm{mg} /$ day) to that of allopurinol $300 \mathrm{mg} /$ day in patients with normal renal function and $200 \mathrm{mg} /$ day in patients with CKD in 2269 patients over a 6-month period [39•]. The proportion of patients achieving target $\mathrm{SU}<6.0 \mathrm{mg} /$ dl was 45 and $67 \%$ for febuxostat 40 and $80 \mathrm{mg} /$ day, respectively, and only $42 \%$ for patients on allopurinol. The most common observed side effects included liver function abnormalities, nausea, arthralgia, and rash [37].

\section{Uricolytic drugs}

Uricase catalyzes the oxidation UA into allantoin, the soluble form. Uricase is inactive in humans due to silent mutations of the genes coding for uricase; hence, most of the uric acid needs to be excreted via the kidneys

Pegloticase, a recombinant mammalian uricase, was approved by the FDA in 2009 for the treatment of refractory gout (patients with severe gout intolerance or refractory to allopurinol with $\mathrm{SU}>8 \mathrm{mg} /$ dl) [36•]. The dose approved by the FDA is $8 \mathrm{mg}$ as biweekly or monthly infusions.

The phase 3 studies included 225 patients treated with pegloticase biweekly or monthly versus placebo for 6 months [40]. Forty-seven percent of patients on biweekly pegloticase achieved a target $S U \leq 6 \mathrm{mg} / \mathrm{dl}$. Becker et al. [41] reported the long-term safety of pegloticase in 149 patients, who had received $28 \pm 18$ pegloticase infusions and were followed for a mean duration of $25 \pm 11$ months. Gout attacks and infusion reactions (IR) were the most common adverse events. Ten of the eleven patients with serious infusion reactions had a SU of $>6 \mathrm{mg} / \mathrm{dl}$. When patients consistently had SU of $<6 \mathrm{mg} / \mathrm{dl}$, there was sustained and progressive improvement in tophus resolution and attack incidence. Other common adverse events include nausea, dizziness, and back pain. Given the immunogenicity of the molecule, some patients develop antibodies to pegloticase, usually within the first 2-3 months of drug initiation. These neutralizing antibodies are 
thought to be responsible for the loss of efficacy and infusion reactions leading to rising SU levels [40]. Thus, it is important to monitor SU levels prior to infusions and stop therapy if two SU levels are $>6 \mathrm{mg} / \mathrm{dl}$ while on pegloticase treatment. It is important to check glucose-6phosphate dehydrogenase (G6PD) levels since in G6PD-deficient patients the oxidant load during conversion of urate to allantoin can cause hemolytic anemia [42].

\section{Uricosuric drugs}

Uricosuric drugs are not recommended as first-line ULT [36•] by the ACR TFP unless the patient has contraindications and/or intolerance to XOI. Uricosurics are contraindicated in patients with a prior history of nephrolithiasis and high urinary uric acid levels [36•].

Probenecid is a urate transporter-1 inhibitor (URAT-1) which inhibits tubular reabsorption of oxypurinol, the active metabolite of allopurinol [43]. It is not recommended in patients with GFR $<50 \mathrm{ml} / \mathrm{min} / 1.73 \mathrm{~m}^{2}[36 \bullet]$. Combination of a XOI with a uricosuric drug is recommended by the ACR TFP in patients who have not achieved target SU levels [36•].

Probenecid is used in doses of up to $1 \mathrm{~g} /$ day and is more effective as twice/thrice daily dosing which can raise compliance issues [43]. Probenecid is not recommended in patients with GFR $<50 \mathrm{ml} / \mathrm{min}$ and in patients with history of urolithiasis. If used in such patients, a large amount of water should be consumed and urine should be alkalinized to prevent development of urolithiasis [43].

Benzobramone never received FDA approval because of concerns of liver toxicity. Given the efficacy in underexcretors as demonstrated by Reinders MK et al. [44], it was initially approved in Europe and Asia and it has since been withdrawn in many countries starting in 2003 due to the risk of hepatotoxicity and bone marrow suppression [45]. The hepatic metabolism of benzbromarone by CYP 2C9 and the effects of the parent compound and/or its metabolites on mitochondrial function are thought to be related to its hepatotoxicity.

Losartan, an angiotensin II receptor inhibitor, has uricosuric effects via the inhibition of the URAT-1 and glucose transporter (GLUT)-9 [43]. It may be attractive for use in patients with coexistent hypertension.

Fenofibrate, a cholesterol-lowering medication, inhibits URAT-1 and has a mild uricosuric effect. Losartan and fenofibrate in combination with allopurinol $200 \mathrm{mg}$ twice daily has been shown to have a significant hypouricemic effect [46]. It may be attractive for use in patients with coexistent hypertriglyceridemia. 


\section{Investigational drugs}

Lesinurad, a uricosuric drug currently in development [47], inhibits the URAT-1 as well as the OAT4 transporter in the proximal renal tubule leading to uricosuria. Lesinurad has been studied in combination with allopurinol as well as febuxostat. In the CLEAR1 and CLEAR2 (Combining Lesinurad with Allopurinol in Inadequate Responders), both lesinurad 200 and $400 \mathrm{mg}$ in combination with allopurinol had a statistically significant higher proportion of patients reaching the target SU at month 6 compared to allopurinol alone $(p<0.0001)$ [48].

Thus, the potential of combination therapy with lesinurad may provide a future treatment option for gout patients on allopurinol or febuxostat where additional therapy is needed.

Other novel agents such as arhalofenate, ulodesine, and levotofisopam are currently in development [47]. However, lesinurad is the only drug which has completed phase 3 studies.

\section{Non-pharmacological treatment}

Diet and lifestyle modification, although insufficient by themselves, are an important part of the management of gout. Combination of diet and exercise can contribute to a 10-18 \% reduction in SU levels [49]. Eating purine-rich foods increases the uric acid pool, and hence, the ACR TFP recommends that patients with gout limit their consumption of organ meats, seafood, alcohol (defined as $>2$ servings/day for males and $>1$ serving/day for females), and high-fructose corn syrup sweetened beverages. There is evidence to suggest that increasing the consumption of low-fat dairy products and foods rich in vitamin C can reduce SU levels [50, 51], and hence, the ACR TFP [36•] encourages the consumption of low-fat or non-fat dairy products.

\section{Conclusion}

We have shown that gout treatment has advanced greatly in recent years. In this paper, we reviewed treatment of acute and chronic gout, while highlighting the latest American College of Rheumatology guidelines when relevant. Better understanding of gout pathogenesis, new drugs for the treatment of gout today and on the horizon for tomorrow as well as recently published recommendations and guidelines for treatment lead to a growing interest in gout. Thus, in contrast to DrTalbot's views, gout therapeutics are more satisfactory. With more therapeutic options on the horizon, the future looks bright for our gout patients.

\section{Compliance with Ethics Guidelines}

\section{Conflict of Interest}

Sneha Pai and Ashraf Raslan declare that they have no conflict of interest. 
Naomi Schlesinger declares the following: Receipt of a grant from Novartis and consultancy fees from Novartis, Sobi, and BMS; Advisory boards: Novartis, Takeda, and Sobi; and Speakers bureau: Novartis, Takeda.

Human and Animal Rights and Informed Consent

This article does not contain any studies with human or animal subjects performed by any of the authors.

\section{References and Recommended Reading}

Papers of particular interest, published recently, have been

highlighted as

- Of importance

1. Talbot JH. Gout. 2nd ed. New York: Grune \& Stratton Inc; 1964. p. 192-6.

2. Dalbeth N, Lauterio TJ, Wolfe HR. Mechanism of action of colchicine in the treatment of gout. Clin Ther. 2014;36(10):1465-79.

3. $\quad$ Khanna D, Khanna PP, Fitzgerald JD, Singh MK, Bae S, Neogi T, et al. American College of Rheumatology guidelines for management of gout. Part 2: therapy and antiinflammatory prophylaxis of acute gouty arthritis. Arthritis Care Res. 2012;64(10):1447-61.

This publication summarizes the American College of Rheumatology guidelines for the management of acute gout; while providing the reader an in-depth understanding of the various pharmacological options for the treatment and prophylaxis of acute gout.

4. Terkeltaub RA, Furst DE, Bennett K, Kook KA, Crockett RS, Davis MW. High versus low dosing of oral colchicines for early acute gout flare. Arthritis Rheum. 2010;62(4):1060-8.

5. Borstad GC, Bryant LR, Abel MP, Scroggie DA, Harris MD, Alloway JA. Colchicine for prophylaxis of acute flares when initiating allopurinol for chronic gouty arthritis. J Rheumatol. 2004;31(12):2429-32.

6. Tateishi T, Soucek P, Caraco Y, Guengerich FP, Wood AJ. Colchicine biotransformation by human liver microsomes. Identification of CYP3A4 as the major isoform responsible for colchicine demethylation. Biochem Pharmacol. 1997;53:111-6.

7. Kuncl RW, Duncan G, Watson D, Anderson K, Rogawski MA, Peper M. Colchicine myopathy and neuropathy. N Engl J Med. 1987;316(25):1562-8.

8. van Durme CMPG, Wechalekar MD, Buchbinder R, Schlesinger N, van der Heijde D, Landewé RBM. Nonsteroidal anti-inflammatory drugs for acute gout. Cochrane Database Syst Rev published online: 16 Sep 2014 | doi: 10.1002/14651858.CD010120.pub2

9. Janssens HJ, Janssen M, van de Lisdonk EH, van Riel PL, van Weel C. Use of oral prednisolone or naproxen for the treatment of gout arthritis: a double-blind randomised equivalence trial. Lancet. 2008;371(9627):1854-60. This double blind randomized trial demonstrated the equivalent efficacy of oral prednisone (35 mg/day) and naproxen (500 mg twice daily) for the initial treatment of acute gout over 5 days.

10. Man CY, Cheung IT, Cameron PA, Rainer TH. Comparison of oral prednisolone/paracetamol and oral indomethacin/paracetamol combination therapy in the treatment of acute gout like arthritis: a doubleblind, randomized, controlled trial. Ann Emerg Med. 2007;49(5):670-7.

11. Alloway JA, Moriarty MJ, Hoogland YT, Nashel DJ. Comparison of triamcinolone acetonide with indomethacin in the treatment of acute gouty arthritis. J Rheumatol. 1993;20(1):111-3.

12. Chen D, Tanjong Ghogomu E, Schlesinger N. Adrenocorticotropic hormone for acute gout. Review: Cochrane Database Syst Rev 2009

13. Daoussis D, Andonopoulos AP. Adrenocorticotropic hormone: a powerful but underappreciated therapeutic tool for acute crystal induced arthritis? World J Rheumatol. 2013;3(2):6-8.

14. Martinon F, Pétrilli V, Mayor A, Tardivel A, Tschopp J. Gout-associated uric acid crystals activate the NALP3 inflammasome. Nature. 2006;440(7081):237-41.

This publication provides an understanding of the molecular processes involved in the pathogenesis of gout; describing the key role of the inflammasome in gout and other autoinflammatory diseases.

15. So A, De Smedt T, Revaz S, Tschopp J. A pilot study of IL-1 inhibition by anakinra in acute gout. Arthritis Res Ther. 2000;9(2):R28.

16. Cho M, Ghosh P, Hans G, Rhiannon J, Gardner GC, Simkin PA. Treatment of acute gouty arthritis in complex hospitalized patients with anakinra. Arthritis Care Res (Hoboken). 2013;65(8):1381-4.

17. Terkeltaub R, Sundy JS, Schumacher HR, Murphy F, Bookbinder S, Biedermann S, et al. The IL-1 inhibitor rilonacept in treatment of chronic gouty arthritis: results of a placebo-controlled, monosequence crossover, nonrandomized, single-blind pilot study. Ann Rheum Dis. 2009;68(10):1613-7.

18. Investor teleconference: June 9, 2010. (http://files. shareholder.com/downloads/REGN/ 941483174x0x380925/e99d3c78-f180-4597-a98dfc1ce20abe49/REGN_Rilonacept_Call_Presentation. pdf.) 
19. So A, De Meulemeester M, Pikhlak A, et al. Canakinumab for treatment of acute attacks in difficult-to-treat gouty arthritis. Arthritis Rheum. 2010;62(10):3064-76.

20. Schlesinger N, Baker DG, Beutler AM, Rull M, Hoffman BI, Schumacher Jr HR. Local ice therapy during bouts of acute gouty arthritis. J Rheumatol. 2002;29:331-4.

21. Schlesinger N, Thiele RG. The pathogenesis of bone erosions in gouty arthritis. Ann Rheum Dis.

2010;69(11):1907-12.

22. Wortmann RL, Macdonald PA, Hunt B, Jackson RL. Effect of prophylaxis on gout attacks after the initiation of urate-lowering therapy: analysis of data from three phase III trials. Clin Ther. 2010;32(14):2386-97.

23. Sundy JS, Becker MA, Baraf HS, Barkhuizen A, Moreland LW, Huang W, et al. for the Pegloticase Phase 2 Study Investigators. Reduction of plasma urate levels following treatment with multiple doses of pegloticase (polyethylene glycol-conjugated uricase) in patients with treatment-failure gout: results of a phase II randomized study. Arthritis Rheum. 2008;58(9):2882-91.

24. Paulus HE, Schlosstein LH, Godfrey RG, Klinenberg JR, Bluestone R. Prophylactic colchicine therapy of intercritical gout. A placebo-controlled study of probenecid-treated patients. Arthritis Rheum. 1974;17(5):609-14.

25. Wertheimer AI, Davis MW, Lauterio TJ. A new perspective on the pharmacoeconomics of colchicine. Curr Med Res Opin. 2011;27(5):931-7.

26. Zhang W, Doherty M, Bardin T, Pascual E, Barskova V, Conaghan $\mathrm{P}$, et al. EULAR evidence based recommendations for gout. Part II: management. Report of a task force of the EULAR Standing Committee for International Clinical Studies Including Therapeutics (ESCISIT). Ann Rheum Dis. 2006;65:1312-24.

27. COLCRYS (colchicine, USP) Tablets [prescribing information] Updated 3/2014. Accessed online 12/12/ 2014. https://www.colcrys.com/safety-and-side-effects. aspx

28. Peterson DM. Nonsteroidal anti-inflammatory drugs and colchicine to prevent gout attack during early urate-lowering therapy: perspectives on alternative therapies and costs. J Pain Palliat Care Pharmacother. 2010;24(4):402-4.

29. Keenan RT, O'Brien WR, Lee KH, Crittenden DB, Fisher MC, Goldfarb DS, et al. Prevalence of contraindications and prescription of pharmacologic therapies for gout. Am J Med. 2011;124(2):155-63.

30. Schlesinger N. Treatment of chronic gouty arthritis: it's not just about urate-lowering therapy. Semin Arthritis Rheum. 2012;42(2):155-65.

31. Schumacher Jr HR, Sundy JS, Terkeltaub R, Knapp HR, Mellis SJ, Stahl N, et al. Rilonacept (IL-1 Trap) in the prevention of acute gout flares during initiation of urate-lowering therapy: results of a phase 2 clinical trial. Arthritis Rheum. 2012;64(3):876-84.

32.• Schlesinger N, Mysler E, Lin HY, De Meulemeester M, Rovensky J, Arulmani U, et al. Canakinumab reduces the risk of acute gouty arthritis flares during initiation of allopurinol treatment: results of a double-blind, randomised study. Ann Rheum Dis. 2011;70(7):126471. This publication reports safety and efficacy of canakinumab versus colchicine in a randomized controlled trial demonstrating superior efficacy of one subcutaneous injection of canakinumab in doses $>50 \mathrm{mg}$ or weekly injections when compared to oral colchicine $0.5 \mathrm{mg}$ given once daily for acute gout prophylaxis.

33. Zhang Y, Neogi T, Chen C, Chaisson C, Hunter DJ, Choi HK. Cherry consumption and the risk of recurrent gout attacks. Arthritis Rheum. 2012;64(12):4004-11.

34. Di Giovine FS, Malawista SE, Nuki G, Duff GW. Interleukin 1 (IL 1) as a mediator of crystal arthritis. Stimulation of T cell and synovial fibroblast mitogenesis by urate crystal induced IL 1. J Immunol. 1987; 138(10):3213-8.

35. Schlesinger N, Rabinowitz R, Schlesinger M. Pilot studies of cherry juice concentrate for gout flare prophylaxis. J Arthritis. 2012;1:1. doi:10.4172/jah s. 1000101.

36. Khanna D, Fitzgerald JD, Khanna PP, Bae S, Singh MK, Neogi T, et al. American College of Rheumatology guidelines for management of gout. Part 1: systematic nonpharmacologic and pharmacologic therapeutic approaches to hyperuricemia. Arthritis Care Res (Hoboken). 2012;64(10):1431-46.

This publication summarizes the American College of Rheumatology guidelines for the management of hyperuricemia and provides the reader an understanding of the pharmacological and non-pharmacological interventions for treatment of hyperuricemia.

37. Crittenden DB, Pillinger MH. New therapies for gout. Annu Rev Med. 2013;64:325-37.

38. Seth R, Kydd AS, Buchbinder R, Bombardier C, Edwards CJ. Allopurinol for chronic gout. Cochrane Database Syst Rev. 2014;10

39. Becker MA, Schumacher HR, Espinoza LR, Wells AF, MacDonald P, Lloyd E, et al. The urate-lowering efficacy and safety of febuxostat in the treatment of the hyperuricemia of gout: the CONFIRMS trial. Arthritis Res Ther. 2010;12(2):R63.

This publication reports the results of the randomized controlled trial - CONFIRMS, which established the urate lowering efficacy of febuxostat versus allopurinol in gout patients with and without renal impairment.

40. Sundy JS, Baraf HS, Yood RA, Edwards NL, Gutierrez Urena SR, Treadwell EL, et al. Efficacy and tolerability of pegloticase for the treatment of chronic gout in patient's refractory to conventional treatment: two randomized controlled trials. JAMA. 2011;306(7):711-20.

41. Becker MA, Baraf HS, Yood RA, Dillon A, Vazquez-Mellado J, Ottery FD, et al. Long-term safety of pegloticase in chronic gout refractory to conventional treatment. Ann Rheum Dis. 2013;72(9):1469-74. 
42. Shannon JA, Cole SW. Pegloticase: a novel agent for treatment-refractory gout. Ann Pharmacother. 2012;46(3):368-76.

43. Bach $\mathrm{MH}$, Simkin PA. Uricosuric drugs: the once and future therapy for hyperuricemia? Curr Opin Rheumatol. 2014;26(2):169-75.

44. Reinders MK, Haagsma C, Jansen TL, van Roon EN, Delsing J, van de Laar MA, et al. A randomised controlled trial on the efficacy and tolerability with dose escalation of allopurinol 300-600 mg/day versus benzbromarone 100-200 mg/day in patients with gout. Ann Rheum Dis. 2009;68(6):892-7.

45. Liver toxicity of benzbromarone. National Institute of Health. Updated 5/2014. http://livertox.nih.gov/ Benzbromarone.htm. Accessed online on 12/12/2014.

46. Takahashi S, Moriwaki Y, Yamamoto T, Tsutsumi Z, Ka $\mathrm{T}$, Fukuchi M. Effects of combination treatment using antihyperuricaemic agents with fenofibrate and/or losartan on uric acid metabolism. Ann Rheum Dis. 2003;62(6):572-5.
47. Edwards NL, So A. Emerging therapies for gout. Rheum Dis Clin North Am. 2014;40(2):375-87.

48. Saag, KG, Adler S, Bhakta N, Fung M, Kopicko J, Storgard C, BardinT. Lesinurad, A novel selective uric acid reabsorption inhibitor, in two phase iii clinical trials: combination study of lesinurad in allopurinol standard of care inadequate responders (CLEAR 1 and 2). ACR 2014;L10

49. Singh JA, Reddy SG, Kundukulam J. Risk factors for gout and prevention: a systematic review of the literature. Curr Opin Rheumatol. 2011;23(2):192-202.

50. Zgaga L, Theodoratou E, Kyle J, Farrington SM, Agakov $\mathrm{F}$, Tenesa A, et al. The association of dietary intake of purine-rich vegetables, sugar-sweetened beverages and dairy with plasma urate, in a cross-sectional study.

PLoS One. 2012;7(6).

51. Dalbeth N, Palmano K. Effects of dairy intake on hyperuricemia and gout. Curr Rheumatol Rep. 2011;13(2):132-7. 